\title{
Wild Hyperbolic Sets, Yet no Chance for the Coexistence of Infinitely Many KLUS-Simple Newhouse Attracting Sets ${ }^{\star}$
}

\author{
Helena E. Nusse ${ }^{1}$ and Laura Tedeschini-Lalli ${ }^{2}$ \\ ${ }^{1}$ University of Maryland, Institute for Physical Science and Technology, College Park, \\ MD 20742, USA and Rijksuniversiteit Groningen, Fac. Economische Wetenschappen, WSN- \\ gebouw, Postbus 800, NL-9700 AV Groningen, The Netherlands \\ 2 Dipartimento di Matematica "G. Castelnuovo," Università di Roma "La Sapienza," P. le \\ Aldo Moro 5, I-00185 Rome, Italy
}

Received February 10, 1990

\begin{abstract}
The phenomenon 'of the coexistence of infinitely many sinks for two dimensional dissipative diffeomorphisms is a result due to Newhouse [Ne1, Ne2]. In fact, for each parameter value at which a homoclinic tangency is formed nondegenerately, there exist intervals in the parameter space containing dense sets of parameter values for which there are infinitely many coexisting sinks (Robinson $[\mathrm{R}])$. The structure of the sinks constructed by Newhouse is limited. "Simple" Newhouse parameter values are values at which there are infinitely many sinks having some special well defined property concerning the structure. A result due to Tedeschini-Lalli and Yorke [TY] says that the Lebesgue measure of the set of simple Newhouse parameter values is zero when the tangencies are due to the standard "affine" horseshoe map. It is argued in [TY] and [PR] that a more general derivation of this measure zero result would be desirable. The main result of this paper is that the Lebesgue measure of the set of KLUS-simple parameter values (including the simple Newhouse parameter values) is zero for saddle hyperbolic basic sets forming tangencies.
\end{abstract}

\section{Introduction}

Let $M$ be a smooth 2-dimensional compact manifold, and let $\left\{F_{\mu}\right\}_{\mu \in I} \subset \operatorname{Diff}^{3}(M)$ be a one parameter family of dissipative $C^{3}$-diffeomorphisms so that for each $\mu \in I$ the diffeo $F_{\mu}$ maps $M$ to itself, where $I \subset \mathbb{R}$ is some compact interval. For $\mu \in I$, a point $q \in M$ is called a sink (respectively a saddle) of $F_{\mu}$ if $q$ is a periodic point, say

* Research in Part supported by Gruppo Nazionale per la Fisica Matematica, CNR 
$F_{\mu}^{p}(q)=q$, and the eigenvalues $\sigma$ and $\lambda$ of the Jacobian matrix $D F_{\mu}^{p}(q)$ satisfy $|\sigma|<1$, $|\lambda|<1$ (respectively $\sigma$ and $\lambda$ are both real and $0<|\sigma|<1<|\lambda|$ ). If the period $p$ of a sink (saddle) is minimal, then the sink (saddle) is called a p-sink (p-saddle).

We say $\mu_{0} \in I$ is a nondegenerate homoclinic tangency value for $\left\{F_{\mu}\right\}_{\mu \in I}$ if this family creates, for a $p$-saddle $q_{\mu} \in \Lambda_{\mu}$, a nondegenerate homoclinic intersection at $x=r_{0}, \mu=\mu_{0}$, that is (see also Sect. 2), there exist $\varepsilon>0$, and subarcs $\gamma_{\mu}^{s} \subset W^{s}\left(q_{\mu} ; F_{\mu}\right)$ and $\gamma_{\mu}^{u} \subset W^{u}\left(q_{\mu} ; F_{\mu}\right)$ varying differentiably with $\mu$ such that: (1) for $\mu \in\left[\mu_{0}-\varepsilon, \mu_{0}\right)$ the segments $\gamma_{\mu}^{s}$ and $\gamma_{\mu}^{u}$ do not intersect; (2) for $\mu=\mu_{0}$ the segments $\gamma_{\mu}^{s}$ and $\gamma_{\mu}^{u}$ are tangent at $r_{0}$, and the intersection at $r_{0}$ is of order two (equivalently, the tangency is of order one); (3) for $\mu \in\left(\mu_{0}, \mu_{0}+\varepsilon\right]$ the segment $\gamma_{\mu}^{u}$ crosses $\gamma_{\mu}^{s}$ from one side to the other and returns to the original side. Hence, when $\mu_{0}$ is a nondegenerate homoclinic tangency value, then for $\mu>\mu_{0}$ there are two new transverse intersections and a Smale horseshoe for some iterate of the map.

The following surprising result is mainly due to Newhouse [ $\mathrm{Ne} 1, \mathrm{Ne} 2]$; Robinson [R] showed that Newhouse's result can also be formulated for one parameter family of diffeomorphisms, and we will use this latter approach.

A parameter value $\mu \in I$ is called a Newhouse parameter value if $F_{\mu}$ has infinitely many coexisting sinks. Let $\mu_{0} \in I$ be a nondegenerate tangency value such that the stable manifold and unstable manifold of a 1 -saddle of $F_{\mu_{0}}$ have a nontransversal intersection at $r_{0}$. Then the following holds (see [ $\mathrm{Ne} 1, \mathrm{Ne} 2$, and $\left.\mathrm{R}\right]$ for details):

(1) For each $n$ sufficiently large there exists a $p_{n}-\operatorname{sink} r_{n}$ of $F_{\mu(n)}$ such that $p_{n} \rightarrow \infty$, $r_{n} \rightarrow r_{0}$ and $\mu(n) \rightarrow \mu_{0}$ as $n \rightarrow \infty$; (2) for every $\varepsilon>0$ there exists an interval $H_{\varepsilon} \subset\left[\mu_{0}-\varepsilon, \mu_{0}+\varepsilon\right)$ in which the set of Newhouse parameter values is residual.

Part (2) follows from (1) in [ $\mathrm{Ne} 2, \mathrm{R}]$ by proving that arbitrarily close to $\mu_{0}$, for which there is a tangency at $r_{0}$, there is a wild hyperbolic set, that is, a hyperbolic set $\Delta_{\mu}$ displaying tangencies for each $\mu$ in an interval arbitrarily close to $\mu_{0}$.

It is important to know the answer to the question "What is the Lebesgue measure of such a residual set (that is, countable intersection of open dense sets) of Newhouse parameter values?". In [PR] it is mentioned that such a result might give insight into the well known problem of whether the standard Hénon map (parameter values 1.4 and 0.3 ) has a strange attractor or not. In full generality, this is a difficult problem. A first approach to attack this problem was undertaken by Tedeschini-Lalli and Yorke [TY]. They considered the so-called simple Newhouse parameter values (Newhouse parameter values for which the sinks have some limited structure), and obtained the result that the Lebesgue measure of the set of simple Newhouse parameter values for the standard ("affine") horseshoe map is zero. For this map, Wang [W] improved the result and obtained that the Hausdorff dimension of the set of simple Newhouse parameter values is positive (and smaller than one).

A very natural question is: "What can be said about the Lebesgue measure of some well-defined subset of the Newhouse parameter values for the family $\left\{F_{\mu}\right\}_{\mu \in I}$ when for all $\mu \in I, F_{\mu}$ has a zero dimensional hyperbolic basic set $\Lambda_{\mu}$ varying differentiably with $\mu$ and creating tangencies near $\mu_{0}$ ?". We will deal with this problem.

In this paper, we give a definition of KLUS-simple parameter values based on the structure of orbits that has been described in [TY]. We would like to emphasize that a simple Newhouse parameter value is a KLUS-simple parameter value. The main result of this paper is that the Lebesgue measure of the set of KLUS-simple 
parameter values is zero. We argue that it is sufficient to assume that $F$ is of class $C^{2}$. In Sect. 4 we also organize a hierarchy of non-simple sinks whose infinite coexistence has still probability zero.

\section{Statement of the Result}

Let $M$ be a two dimensional compact smooth manifold. We write $\operatorname{Diff}^{3}(M)$ for the class of $C^{3}$ diffeomorphisms from $M$ to itself.

Let $F \in \operatorname{Diff}^{3}(M)$. A subset $\Lambda$ of $M$ is hyperbolic if $\Lambda$ is compact, invariant, and there is a continuous splitting $T_{A} M=E_{A}^{s} \oplus E_{A}^{u}$ of the tangent bundle $T_{A} M$, a constant $0<\xi<1$ and a Riemannian norm $\|\cdot\|$ on TM such that for each $x \in \Lambda$ (1) $T_{x} F\left(E_{x}^{s}\right)=E_{F(x)}^{s}, T_{x} F\left(E_{x}^{u}\right)=E_{F(x)}^{u} ;$ (2) $\left\|T_{x} F\left(v^{s}\right)\right\| \leqq \xi\left\|v^{s}\right\|$ for all $v^{s} \in E_{x}^{s}$, $\left\|T_{x} F^{-1}\left(v^{u}\right)\right\| \leqq \xi\left\|v^{u}\right\|$ for all $v^{u} \in E_{x}^{u}$, where $T_{x} F$ denotes the derivative of $F$ at $x$. A set $\Lambda \subset M$ is a hyperbolic basic set if (1) $\Lambda$ is hyperbolic, (2) $F$ is transitive on $\Lambda$ (that is, there is an orbit of $F$ which is dense in $\Lambda$ ), and (3) there exists an open neighborhood $U$ of $\Lambda$ such that $\Lambda=\bigcap_{n \in \mathbb{Z}} F^{n}(U)$. A set $\Lambda \subset M$ is a saddle basic set if $\Lambda$ is a hyperbolic basic set for which $\operatorname{dim}\left(E_{A}^{s}\right)=\operatorname{dim}\left(E_{A}^{u}\right)=1$. Note that each periodic point with minimum period $p$ in a saddle basic set is a $p$-saddle.

Let $\rho$ denote the induced distance function on $M$. For a set $Y \subset M$ the stable set of $Y$ is $W^{s}(Y ; F)=\left\{x \in M: \rho\left(F^{n}(x), F^{n}(Y)\right) \rightarrow 0\right.$ as $\left.n \rightarrow \infty\right\}$ and the unstable set of $Y$ is $W^{u}(Y ; F)=\left\{x \in M: \rho\left(F^{n}(x), F^{n}(Y)\right) \rightarrow 0\right.$ as $\left.n \rightarrow-\infty\right\}$. For a saddle basic set $\Lambda$ of $F$ one has the stable set $W^{s}(\Lambda ; F)=\bigcup_{x \in A} W^{s}(x ; F)$, (respectively the unstable set $\left.W^{u}(\Lambda ; F)=\bigcup_{x \in \Lambda} W^{u}(x ; F)\right)$ is the union of the stable manifolds (respectively the unstable manifolds) of all the elements of $\Lambda$. A neighborhood $U$ of a saddle hyperbolic basic set $\Lambda$ is called a saddle isolating neighborhood for $\Lambda$ if

$$
\Lambda=\bigcap_{n \in \mathbb{Z}} F^{n}(U) \text {, and }
$$

$$
F\left(W^{u}(\Lambda ; F) \cap \partial U\right) \cap \operatorname{Clos} U=\varnothing .
$$

In this case, a saddle basic set is also known as a zero dimensional hyperbolic basic set.

Let $I \subset \mathbb{R}$ denote a compact interval with nonempty interior. We consider the one parameter family $\{F(\cdot ; \mu): \mu \in I\} \subset \operatorname{Diff}^{3}(M)$. For each $\mu \in I$ we write $F_{\mu}=F(\cdot ; \mu)$, and we assume (1) $F_{\mu}$ maps $M$ into itself, (2) the absolute value of the Jacobian of $F_{\mu}$ at $x$ is less than one for each $x \in M$.

For an open neighborhood $U$ of a saddle basic set $\Lambda_{\mu}$ of $F_{\mu}$, where $\mu \in I$, we write

$$
\begin{aligned}
& W_{\mu}^{u}(U)=\bigcap_{n \geqq 0} F_{\mu}^{n}(U) ; \\
& W_{\mu}^{s}(U)=\bigcap_{n \geqq 0} F_{\mu}^{-n}(U) ;
\end{aligned}
$$

and for each positive integer $k$ we write

$$
\begin{aligned}
& W_{\mu ; k}^{u}(U)=\bigcup_{0 \leqq n \leqq k} F_{\mu}^{n}\left(W_{\mu}^{u}(U)\right) ; \\
& W_{\mu ; k}^{s}(U)=\bigcup_{0 \leqq n \leqq k} F_{\mu}^{-n}\left(W_{\mu}^{s}(U)\right) .
\end{aligned}
$$


For each $\mu \in I$ let the maps $g_{1 ; \mu}, g_{2 ; \mu}: \mathbb{R} \rightarrow \mathbb{R}$ be defined by

$$
g_{1 ; \mu}(y)=0, \quad g_{2 ; \mu}(y)=y^{2} .
$$

Let $H \subset I$ be some interval, and let $\kappa: H \rightarrow \operatorname{Diff}^{3}(M)$ defined by $\kappa(\mu)=F_{\mu}$ be a $C^{1}$ curve such that

(A1) For each $\mu \in H$ there exists a saddle basic set $\Lambda_{\mu}$ for $F_{\mu}$ varying differentiably with $\mu$.

(A2) There exists an open neighborhood $U$ in $M$ which is a saddle isolating neighborhood for $\Lambda_{\mu}$ for all $\mu \in H$.

(A3) There exist a smooth open ball $S$ and $C^{3}$ coordinates $\varphi: S \rightarrow \mathbb{R}^{2}$ such that

(a) $U \cap S=\varnothing$;

(b) there exist positive integers $K$ and $L$ such that if $x \in W_{\mu ; K}^{u}(U) \cap W_{\mu ; L}^{s}(U) \cap S$, and if there exists $q \in \Lambda$ so that $W^{u}\left(q ; F_{\mu}\right)$ and $W^{s}\left(q ; F_{\mu}\right)$ are not transversal at $x$, then there exist segments $\gamma_{\mu}^{s} \subset W^{s}\left(q ; F_{\mu}\right), \gamma_{\mu}^{u} \subset W^{u}\left(q ; F_{\mu}\right)$ both including $x$ for which

(b1) $\varphi(x)$ is near $(0,0)$;

(b2) $\varphi\left(\gamma_{\mu}^{s}\right) \subset\left\{\left(z_{1}, z_{2}\right): z_{2}=\psi_{1 ; \mu}\left(z_{1}\right), \psi_{1 ; \mu}\right.$ is $C^{2}$ close to $\left.g_{1 ; \mu}\right\}$;

(b3) $\varphi\left(\gamma_{\mu}^{u}\right) \subset\left\{\left(z_{1}, z_{2}\right): z_{2}=\psi_{2 ; \mu}\left(z_{1}\right), \psi_{2 ; \mu}\right.$ is $C^{2}$ close to $\left.g_{2 ; \mu}\right\}$;

(b4) $\frac{\partial}{\partial \mu} y^{*}(\mu)<0$, where $y^{*}(\mu)=\min \left\{z_{2}:\left(z_{1}, z_{2}\right) \in \varphi\left(\gamma_{\mu}^{u}\right)\right\}$.

We call an interval $H \subset I$ a KLUS interval if the curve $\kappa$ has the above properties. KLUS intervals are introduced to describe tangencies outside isolated neighborhoods of saddle basic sets, making the required estimates in the proofs more transparent.

Let $H$ be a KLUS interval; for $\mu \in H$ we say a $p$-sink $q_{\mu} \in S$ is a KLUS sink for $F_{\mu}$ if the orbit $\operatorname{Orb}\left(q_{\mu}\right)$ of $q_{\mu}$ satisfies:

(1) $\operatorname{orb}\left(q_{\mu}\right) \cap S=\left\{q_{\mu}\right\}$; (2) $\operatorname{Orb}\left(q_{\mu}\right) \subset \bigcup_{-L \leqq n \leqq k} F_{\mu}^{n}(U)$; and

(3) $\operatorname{Orb}\left(q_{\mu}\right) \cap U$ consists of at least $p-(K+L)$ elements. Hence (by definition) the number of iterates of a KLUS sink that are not contained in $U$ is at most $K+L$, for all parameter values in a KLUS interval.

The family $\left\{F_{\mu}\right\}_{\mu \in I}$ nondegenerately creates a homoclinic intersection at $x=r_{0}$, $\mu=\mu_{0}$ for a $p$-saddle $q_{\mu} \in \Lambda_{\mu}$, if there exists $\varepsilon>0$, and if there are one-dimensional disks $\gamma_{\mu}^{s} \subset W^{s}\left(q_{\mu} ; F_{\mu}\right)$ and $\gamma_{\mu}^{u} \subset W^{u}\left(q_{\mu} ; F_{\mu}\right)$ varying differentiably with $\mu$, such that:

(1) $\gamma_{\mu}^{s} \cap \gamma_{\mu}^{u}=\varnothing$ for $\mu \in\left[\mu_{0}-\varepsilon, \mu_{0}\right)$;

(2) there are $C^{3}$ local coordinates $\{(x, y)\}$ near $r_{0}$ depending differentiably on $\mu$, and parametrizations $\left\{\left(x_{\mu}^{s}(\tau), y_{\mu}^{s}(\tau)\right):|\tau| \leqq \delta\right\}$ of $\gamma_{\mu}^{s}$ and $\left\{\left(x_{\mu}^{u}(\tau), y_{\mu}^{u}(\tau)\right):|\tau| \leqq \delta\right\}$ of $\gamma_{\mu}^{u}$ for some $\delta>0$ such that

(2a) $\left(x_{\mu_{0}}^{s}(0), y_{\mu_{0}}^{s}(0)\right)=(0,0)=\left(x_{\mu_{0}}^{u}(0), y_{\mu_{0}}^{u}(0)\right)$;

(2b) $y_{\mu_{0}}^{s}(\tau)=0$ for each $\tau \in[-\delta, \delta]$;

(2c) sign $y_{\mu}^{u}(\delta)=\operatorname{sign} y_{\mu}^{u}(-\delta)=-\operatorname{sign} y_{\mu}^{u}(\hat{\tau})$ for some $|\hat{\tau}|<\delta$, where $\hat{\tau}$ might depend on $\mu$, for $\mu \in\left(\mu_{0}, \mu_{0}+\varepsilon\right]$;

(3) $\gamma_{\mu_{0}}^{s}$ and $\gamma_{\mu_{0}}^{u}$ have a tangency of order one at $r_{0}$, that is, $y_{\mu_{0}}^{u}(0)=0=\frac{d}{d \tau} y_{\mu_{0}}^{u}(0)$ and $\frac{d^{2}}{d \tau^{2}} y_{\mu_{0}}^{u}(0) \neq 0$; 
(4) the extremum value $y^{*}(\mu)$ of $y_{\mu}^{u}$ on the interval $[-\delta, \delta]$ satisfies $\frac{d}{d \mu} y^{*}\left(\mu_{0}\right) \neq 0$.

Let $I_{*} \subset I$ be a given interval and assume that $U$ is a saddle isolating neighborhood for the saddle basic set $\Lambda_{\mu}$, where $\mu \in I_{*}$. Assume that the family $\left\{F_{\mu}\right\}_{\mu \in I}$ creates for a $p$-saddle $q_{\mu} \in \Lambda_{\mu}$ a nondegenerate homoclinic intersection at $x=r_{0} \in M \backslash \operatorname{Clos} U, \mu=\mu_{0} \in \operatorname{Int} I_{*}$.

Proposition. There exist an open ball $S$ containing $r_{0}$, positive integers $K$ and $L$, and $\delta_{\mu_{0}}>0$ such that $\left[\mu_{0}-\delta_{\mu_{0}}, \mu_{0}+\delta_{\mu_{0}}\right]$ is a KLUS interval.

For $\mu \in I$ and saddle basic set $\Lambda_{\mu}$ of $F_{\mu}$, a point $z_{\mu} \in M \backslash \Lambda_{\mu}$ is called a KLUS-simple Newhouse sink if there exist an open ball $S$ including $z_{\mu}$, an open saddle isolating neighborhood $U$ of $\Lambda_{\mu}$ for which $U \cap S=\varnothing$, positive integers $K$ and $L$, and an interval $H \subset I$ including $\mu$ such that the following hold: (1) $z_{\mu}$ is a KLUS sink for $F_{\mu}$, and (2) $H$ is a KLUS interval.

For $\mu \in I$, a set $\Gamma_{\mu} \subset M$ is called an attracting set of $F_{\mu}$ if (1) $\Gamma_{\mu}$ is compact and invariant, and (2) there exists an open neighborhood $V_{\mu}$ of $\Gamma_{\mu}$ such that $\lim _{n \rightarrow \infty} \rho\left(F_{\mu}^{n}(x), \Gamma_{\mu}\right)=0$ for every $x \in V_{\mu} ; \Gamma_{\mu}$ is called an attractor if $(1) \Gamma_{\mu}$ is an attracting set and (2) $F_{\mu}$ has a dense orbit on $\Gamma_{\mu}$.

Let for $\mu_{0} \in I$, a point $a_{\mu_{0}}^{n}$ be a periodic point of minimum period $n$. Let $I_{*} \subset I$ be the maximal interval including $\mu_{0}$ such that for $\mu \in I_{*}$ the point $a_{\mu}^{n}$ is a periodic point with minimum period $n$ and $a_{\mu}^{n}$ varies differentiably with $\mu$. For each $\mu_{*} \in I_{*}$ we call $\left\{a_{\mu}^{n}: \mu \in I_{*}\right\}$ the path of $a_{\mu_{*}}^{n}$

For $\mu \in I$, a set $\Gamma_{\mu}$ is called a p-attracting set if (1) $\Gamma_{\mu}$ is an attracting set for $F_{\mu}^{p}$, and (2) for every $k, 1 \leqq k \leqq p-1, \Gamma_{\mu}$ is not an attracting set for $F_{\mu}^{k}$; we call the number $p$ the period of the attracting set $\Gamma_{\mu}$. $\Gamma_{\mu}$ is called a KLUS-simple attracting set if there exists $p \in \mathbb{N}$ such that (1) $\Gamma_{\mu}$ is a $p$-attracting set, (2) $\Gamma_{\mu}$ includes a point that is on the path of some KLUS-simple $p$-sink, and (3) $\Gamma_{\mu_{*}}$ is a topological horsehoe for some $\mu_{*}>\mu$. For instance, a $p$-sink is both a $p$-attracting set and an attractor. The parameter value $\mu \in I$ is called a KLUS-simple parameter value if $F_{\mu}$ has infinitely many KLUS-simple attracting sets of different period.

Theorem. The Lebesgue measure of the set of KLUS-simple parameter values in I is zero.

Corollary. The set of parameter values in I, for which there are infinitely many KLUS-simple Newhouse sinks, is zero.

We would like to emphasize that having allowed an attracting set to contain several attractors, it might very well be, and indeed is generally the case, that a KLUS-simple attracting set does in turn contain infinitely many attractors. We will discuss in Sect. 4 which of these non-simple attractors can still be included in our approach.

\section{Proofs of the Results}

Let $\left\{F_{\mu}\right\}_{\mu \in I} \subset \operatorname{Diff}^{3}(M)$, and $\rho$ the distance function on $M$ (induced by the Riemannian metric). We assume that for every $\mu \in I$ the set $\Lambda_{\mu}$ is a nontrivial saddle 
basic set for $F_{\mu}$, that is, $\Lambda_{\mu}$ includes more than a periodic orbit, and $\Lambda_{\mu}$ varies differentiably with $\mu$.

3A. Preliminaries. In this subsection, we present some auxiliary results for the one parameter family of diffeomorphisms. Let $\mu \in I$ be arbitrarily fixed. For a nonwandering point $z_{\mu}$ (a point such that for every open neighborhood $V$ of $z_{\mu}$ there exists $n \geqq 1$ for which $\left.F_{\mu}^{n}(V) \cap V \neq \varnothing\right)$ the local stable manifold $W_{\text {loc }}^{s}\left(z_{\mu} ; F_{\mu}\right)$ (respectively local unstable manifold $W_{\text {loc }}^{u}\left(z_{\mu} ; F_{\mu}\right)$ ) of $z_{\mu}$ of size $\beta$ is the set of points $x$ in the stable manifold $W^{s}\left(z_{\mu} ; F_{\mu}\right)$ (respectively unstable manifold $W^{u}\left(z_{\mu} ; F_{\mu}\right)$ ) so that $\rho\left(F_{\mu}^{n}\left(z_{\mu}\right), F_{\mu}^{n}(x)\right) \leqq \beta$ (respectively $\rho\left(F_{\mu}^{-n}\left(z_{\mu}\right), F_{\mu}^{-n}(x)\right) \leqq \beta$ ) for all integers $n \geqq 0$, where $\beta>0$. When the stable or unstable manifold is a curve, we write $W_{\text {loc }}^{\sigma+}\left(z_{\mu} ; F_{\mu}\right)$ and $W_{\text {loc }}^{\sigma-}\left(z_{\mu} ; F_{\mu}\right)$ for the two components of $W_{\text {loc }}^{\sigma}\left(z_{\mu} ; F_{\mu}\right) \backslash\left\{z_{\mu}\right\}$, where $\sigma$ is either $s$ or $u$. The nontrivial saddle basic set $\Lambda_{\mu}$ is called periodic if there exists $m \in \mathbb{N}$ such that $F^{m}$ has no dense orbit on $\Lambda_{\mu}$, and $\Lambda_{\mu}$ is called nonperiodic otherwise. The following results 3-1-3-3, which are rephrased from [NP and PT], say that the structure of saddle basic sets is essentially controlled by finite sets of periodic points.

Proposition 3-1. There exist finite sets $P_{\mu}^{s}$ and $P_{\mu}^{u}$ of periodic points, such that for all $x \in \Lambda_{\mu}$ :

(1) If $x$ is not a limit point of both $W_{\mathrm{loc}}^{u+}\left(x ; F_{\mu}\right) \cap \Lambda_{\mu}$ and $W_{\mathrm{loc}}^{u-}\left(x ; F_{\mu}\right) \cap \Lambda_{\mu}$, then $x$ is in $W^{s}\left(p_{\mu} ; F_{\mu}\right)$ for some $p_{\mu} \in P_{\mu}^{u}$.

(2) If $x$ is not a limit point of both $W_{\mathrm{loc}}^{s+}\left(x ; F_{\mu}\right) \cap \Lambda_{\mu}$ and $W_{\mathrm{loc}}^{s-}\left(x ; F_{\mu}\right) \cap \Lambda_{\mu}$, then $x \in W^{u}\left(p_{\mu} ; F_{\mu}\right)$ for some $p_{\mu} \in P_{\mu}^{s}$.

Proof. For a proof, see Newhouse and Palis [NP].

Palis and Takens [PT] have shown that there exist regions in $M$, whose boundaries are segments in the stable and unstable manifolds of these finite sets of periodic points $P_{s}$ and $P_{u}$, such that these regions when intersected with the saddle basic set $\Lambda$ form a Markov partition of $\Lambda$.

Proposition 3-2. Assume $\Lambda_{\mu}$ is a nontrivial nonperiodic saddle basic set of $F_{\mu}$, and let $z_{\mu} \in \Lambda_{\mu}$ be fixed. Let $P_{\mu}^{s}$ and $P_{\mu}^{u}$ be as in Proposition 3-1. There exist finitely many disjoint regions $R_{i ; \mu}$ being diffeomorphic images of the square $B=[-1,1] \times[-1,1]$, say $R_{i ; \mu}=h_{i ; \mu}(B), 1 \leqq i \leqq N$, for some $N \in \mathbb{N}$, and $a$ segment $I_{\mu}^{u} \subset W^{u}\left(z_{\mu} ; F_{\mu}\right)$ such that:

(1) $\Lambda_{\mu} \cap R_{i ; \mu} \neq \varnothing$ for all $i$;

(2) $\Lambda_{\mu} \subset \bigcup_{i=1}^{N} R_{i ; \mu}$;

(3) $F_{\mu}\left(\partial_{s} R_{i ; \mu}\right) \subset \sum_{j=1}^{N} \partial_{s} R_{j ; \mu}$ and $F_{\mu}^{-1}\left(\partial_{u} K_{i ; \mu}\right) \subset \bigcup_{j=1}^{N} \partial_{u} R_{j ; \mu}$, where $\partial_{s} R_{i ; \mu}=h_{i ; \mu}(\{(x, y)$ : $|x|=1,-1 \leqq y \leqq 1\})$ respectively $\partial_{u} R_{i ; \mu}=h_{i ; \mu}(\{(x, y):-1 \leqq x \leqq 1,|y|=1\})$ are segments in the stable set $W^{s}\left(P_{\mu}^{u} ; F_{\mu}\right)$ respectively the unstable set $W^{u}\left(P_{\mu}^{s} ; F_{\mu}\right)$; and (4) for every $i, I_{\mu}^{u} \cap R_{i ; \mu}$ consists of exactly one component and $\partial\left(I_{\mu}^{u} \cap R_{i ; \mu}\right) \subset \bigcup_{j=1}^{N} \partial_{s} R_{j ; \mu}$,
$1 \leqq i \leqq N$.

Proof. For a proof, see Palis ad Takens [PT]. 
From now on, let $\Lambda_{\mu}$ denote a saddle basic set for $F_{\mu}, U$ a saddle isolating neighborhood of $\Lambda_{\mu}$, and the point $z_{\mu} \in \Lambda_{\mu}$, the regions $R_{i ; \mu}, 1 \leqq i \leqq N$, and the segment $I_{\mu}^{u} \subset W^{u}\left(\Lambda_{\mu} ; F_{\mu}\right)$ as in Proposition 3-2. There exist a $C^{1+\alpha}$ stable foliation $\mathfrak{F}_{\mu}^{s}$ on a neighborhood $V_{\Lambda ; \mu}^{s}$, respectively a $C^{1+\alpha}$ unstable foliation $\mathfrak{F}_{\mu}^{\mu}$ on a neighborhood $V_{\Lambda ; \mu}^{u}$, of $\Lambda_{\mu}$ for some $\alpha>0$, and it is no restriction to assume that every region $R_{i ; \mu}$ is contained in $V_{\Lambda ; \mu}^{s} \cap V_{A ; \mu}^{u}, 1 \leqq i \leqq N$; see [PT].

Let $\tau_{\mu}: \mathbb{R} \rightarrow W^{u}\left(z_{\mu} ; F_{\mu}\right)$ be a $C^{3}$ parametrization, and define a projection

$$
\pi_{\mu}: \Lambda_{\mu} \rightarrow \bigcup_{i=1}^{N} R_{i ; \mu} \cap I_{\mu}^{u}
$$

by taking in each region $R_{i ; \mu}, 1 \leqq i \leqq N$, the projection along the local stable manifolds into the intersection $I_{\mu}^{u}$ with that region. This projection can be extended from $\Lambda_{\mu}$ to the union of the regions $R_{i ; \mu}$ by projecting along the leaves of the foliation $\mathfrak{F}_{\mu}^{s}$. This extension will be denoted by $\pi_{\text {ext } ; \mu}^{s}$. The following result says that for some iterate $M$, the map $F_{\mu}$ can be viewed as expansive along unstable segments.

Proposition 3-3. There exist a positive integer $M$ and $a C^{1+\alpha}$ map $\varphi_{\mu}$ : $\bigcup_{i=1}^{N} \tau_{\mu}^{-1}\left(I_{\mu}^{u} \cap R_{i ; \mu}\right) \rightarrow \mathbb{R}$ defined by $\varphi_{\mu}(x)=\tau_{\mu}^{-1} \circ \pi_{\text {ext } ; \mu}^{s}{ }^{\circ} F_{\mu}^{M} \circ \tau_{\mu}(x)$ such that $\left|\varphi_{\mu}^{\prime}(x)\right|>1$, for some $\alpha>0$.

Proof. For a proof, see Palis and Takens [PT].

The Escape time $T_{U}\left(x ; F_{\mu}\right)$ of $x \in U$ under $F_{\mu}$ is the minimum value $n$ with the property $F_{\mu}^{n}(x)$ not in $U$. Let $J \subset U$ be a curve segment such that $J$ intersects the stable set $W^{s}\left(\Lambda_{\mu} ; F_{\mu}\right)$ transversally. We define for every integer $k \geqq 1$ :

$$
\begin{aligned}
& A_{k}\left(J ; F_{\mu}\right)=\left\{x \in J: T_{U}\left(x ; F_{\mu}\right) \geqq k\right\}, \\
& D_{k}\left(J ; F_{\mu}\right)=\left\{x \in J: T_{U}\left(x ; F_{\mu}\right)=k\right\} .
\end{aligned}
$$

In particular, $A_{1}\left(J ; F_{\mu}\right)=J$. Hence, for each integer $k \geqq 1$ we have $A_{k+1}\left(J ; F_{\mu}\right)$ is the set of points in $A_{k}\left(J ; F_{\mu}\right)$ whose escape time from $U$ is at least $k+1$; hence, $A_{k+1}\left(J ; F_{\mu}\right)$ is the set of points in $J$ that stay in $U$ under $F_{\mu}^{k}$. The points in $J$ which will stay in $U$ under all iterates will be denoted by $A_{\infty}\left(J ; F_{\mu}\right)$. For every integer $k \geqq 1$ we have:

$$
\begin{aligned}
A_{k}\left(J ; F_{\mu}\right) & =A_{k+1}\left(J ; F_{\mu}\right) \cup D_{k}\left(J ; F_{\mu}\right), \quad \text { and } \\
J & =A_{k+1}\left(J ; F_{\mu}\right) \cup \bigcup_{j=1}^{k} D_{j}\left(J ; F_{\mu}\right),
\end{aligned}
$$

that is, $J$ is the union of the set of points $A_{k+1}\left(J ; F_{\mu}\right)$ whose escape time from $U$ is at least $k+1$, and the set of points $D_{j}\left(J ; F_{\mu}\right)$ whose escape time from $U$ is $j$, where $1 \leqq j \leqq k$. We write

$$
D_{\infty}\left(J ; F_{\mu}\right)=\bigcup_{k=1}^{\infty} D_{k}\left(J ; F_{\mu}\right)
$$

Note that $A_{\infty}\left(J ; F_{\mu}\right)=\bigcap_{k=0}^{\infty} A_{k}\left(J ; F_{\mu}\right)$, and $J=A_{\infty}\left(J ; F_{\mu}\right) \cup D_{\infty}\left(J ; F_{\mu}\right)$.

Denote the length of a segment $E \subset J$ by $\rho(E)$.

Lemma 3-4. Let $R_{\Lambda ; \mu}(U)=\left\{J: J\right.$ component of $\left.W^{u}\left(\Lambda_{\mu} ; F_{\mu}\right) \cap U, J \cap \Lambda_{\mu} \neq \varnothing\right\}$. There exists $\delta_{\Lambda ; \mu}>0$ such that for every $J \in R_{\Lambda ; \mu}(U)$, and for each $k \geqq 1$ : 
(1) Each component of $A_{k}\left(J ; F_{\mu}\right)$ contains components of $D_{k}\left(J ; F_{\mu}\right)$ and $A_{k+1}\left(J ; F_{\mu}\right)$.

(2) Let $A$ be an arbitrarily chosen component of $A_{k}\left(J ; F_{\mu}\right)$. For each component $D$ of $D_{k}\left(J ; F_{\mu}\right) \cap A$, one has $\rho(D) / \rho(A) \geqq \delta_{\Lambda ; \mu}$, and each component $G$ of $A_{k+1}\left(J ; F_{\mu}\right) \cap A$, satisfies $\rho(G) / \rho(A) \geqq \delta_{\Lambda ; \mu}$.

Proof. For the proof, see the proof of the Geometric Lemma II in [NY].

3B. Some KLUS Properties. In this subsection, we present some auxiliary KLUS results. Let $H \subset I$ be a KLUS interval as defined in Sect. 2. Recall that $U$ is a saddle isolating neighborhood of $\Lambda_{\mu}$ for all $\mu \in H, S$ is an open ball disjoint from $U$ and $S \subset F_{\mu}^{-L}(U) \cap F_{\mu}^{K}(U)$ for all $\mu \in H$, and $K, K \in \mathbb{N}$. Obviously, every subinterval in $H$ is a KLUS interval.

Recall that, for every $\mu \in H, V_{\Lambda ; \mu}^{s}$ is a neighborhood of $\Lambda_{\mu}$ on which $\mathfrak{F}_{\mu}^{s}$ is defined. Since each compact interval in $H$ is a KLUS interval, both $\mathfrak{F}_{\mu}^{s}$ and $\mathfrak{F}_{\mu}^{u}$ depend differentiably on $\mu$, and by taking $N$ sufficiently large in Proposition 3-2, we may assume that every region $R_{i ; \mu}$ is contained in $\bigcap_{\mu \in H}\left(V_{\Lambda ; \mu}^{s} \cap V_{\Lambda ; \mu}^{u}\right)$, where $R_{i ; \mu}$ is a region for $F_{\mu}$ as in Proposition 3-2, for all $\mu \in H$. Then we select integer $Q \geqq 1$ such that $\bigcap_{n=-Q}^{Q} F_{\mu}^{n}(U) \subset V_{\Lambda ; \mu}^{s}$, for each $\mu \in H$. Define for each integer $m \geqq Q$ the set $Z_{\Lambda ; \mu}^{[m]}$ as the set of all curve segments $J=[a, b]$ in the closure of $\bigcap_{n=-m}^{m} F_{\mu}^{n}(U)$ so that (1) $\{a, b\} \subset \partial\left(\bigcap_{n=-m}^{m} F_{\mu}^{n}(U)\right)$ and (2) $J$ intersects $W^{s}\left(\Lambda_{\mu} ; F_{\mu}\right)$ transversally. Let for $\mu \in H$ the positive number $\delta_{\Lambda ; \mu}$ be as in Lemma 3-4. For the compact interval $H \subset I$ we define the positive number $\delta_{\Lambda ; H}$ by

$$
\delta_{\Lambda ; H}=\min \left\{\delta_{\Lambda ; \mu}: \mu \in H\right\} .
$$

Proposition 3-5. For every $\mu \in H$, for each $J \in Z_{\Lambda ; \mu}^{[m]}$, and for every integer $k \geqq 1$ :

(1) Each component of $A_{m+k}\left(J ; F_{\mu}\right)$ contains components of $D_{m+k}\left(J ; F_{\mu}\right)$ and $A_{m+k+1}\left(J ; F_{\mu}\right)$.

(2) Let $A$ be an arbitrarily chosen component of $A_{m+k}\left(J ; F_{\mu}\right)$. For each component $D$ of $D_{m+k}\left(J ; F_{\mu}\right) \cap A$, one has $\rho(D) / \rho(A) \geqq \delta_{\Lambda ; H}$, and each component $G$ of $A_{m+k+1}\left(J ; F_{\mu}\right) \cap A$, satisfies $\rho(G) / \rho(A) \geqq \delta_{\Lambda ; H}$.

Proof. Since the projection along the local stable manifolds is of class $C^{1+\alpha}$ for some $\alpha>0$, we obtain the result by applying Lemma 3-4.

From Proposition 3-5 and the assumptions on $F_{\mu}$ we obtain that for each $k \geqq 1$ and all $\mu \in H$, the number of components of $A_{m+k}\left(J ; F_{\mu}\right)$ and that of $D_{m+k}\left(J ; F_{\mu}\right)$ is finite, for all $J \in Z_{A ; \mu}^{[m]}$. Let, for $k \geqq 1$ and $\mu \in H, N\left(A_{k}\right)$ be the number of components of $A_{m+k}\left(J ; F_{\mu}\right)$, and let $N\left(D_{k}\right)$ be the number of components of $D_{m+k}\left(J ; F_{\mu}\right)$, where $J \in Z_{\Lambda ; \mu}^{[m]}$. We write, for each $k \geqq 1$ and $\mu \in H$, the sets $A_{m+k}\left(J ; F_{\mu}\right)$ and $D_{m+k}\left(J ; F_{\mu}\right)$ as the union of their components as follows:

$$
\begin{aligned}
& A_{m+k}\left(J ; F_{\mu}\right)=\sum_{i=1}^{N\left(A_{k}\right)} A_{m+k ; i}\left(J ; F_{\mu}\right), \\
& D_{m+k}\left(J ; F_{\mu}\right)=\bigcup_{i=1}^{N\left(D_{k}\right)} D_{m+k ; i}\left(J ; F_{\mu}\right) .
\end{aligned}
$$


Lemma 3-6. For every $\varepsilon>0$ there exists a positive integer $R$ such that for each $\mu \in H$, for every $J \in Z_{\Lambda ; \mu}^{[m]}$ :

$$
\sum_{k=R}^{\infty} \sum_{j=1}^{N\left(A_{k}\right)}\left[\rho\left(A_{m+k ; j}\left(J ; F_{\mu}\right)\right]^{2}<\varepsilon .\right.
$$

Proof. Let $\varepsilon>0$ be given, and select positive integer $R$ such that $\rho(J)^{2} \cdot\left\{\delta_{\Lambda ; H}\left(2-\delta_{\Lambda ; H}\right)\right\}^{-1} \cdot\left(1-\delta_{\Lambda ; H}\right)^{2 R}<\varepsilon$. Then, for each $\mu \in H$, and for every $J \in Z_{\Lambda ; \mu}^{[m]}$ we have:

$$
\begin{aligned}
& \sum_{k=R}^{\infty} \sum_{j=1}^{N\left(A_{k}\right)}\left[\rho\left(A_{m+k ; j}\left(J ; F_{\mu}\right)\right]^{2}\right. \\
& \quad \leqq \sum_{k=R}^{\infty}\left\{\sum_{j=1}^{N\left(A_{k}\right)}\left[\rho\left(A_{m+k ; j}\left(J ; F_{\mu}\right)\right]\right\}^{2} \leqq \sum_{k=R}^{\infty}\left\{\left(1-\delta_{\Lambda ; H}\right)^{k} \cdot \rho(J)\right\}^{2}\right. \\
& \quad=\rho(J)^{2} \cdot\left\{\delta_{\Lambda ; H}\left(2-\delta_{\Lambda ; H}\right)^{-1} \cdot\left(1-\delta_{\Lambda ; H}\right)^{2 R}<\varepsilon .\right.
\end{aligned}
$$

3C. Rescaling. Assume that the family $\left\{F_{\mu}\right\}_{\mu \in I}$ has for a $p$-saddle $q_{\mu} \in \Lambda_{\mu}$ a nondegenerate homoclinic tangency at $x=r_{0}, \mu=\mu_{0} \in I$. Assume that the eigenvalues $\lambda$ and $\sigma$ of $D F_{\mu_{0}}^{p}\left(q_{\mu_{0}}\right)$ satisfy $\lambda>1>\sigma>0, \sigma \lambda<1$ and some generic condition implying that $C^{2}$-linearlzing cordinates exist in a neighborhood of $q_{\mu}$ for $\mu \in J$, where $J \subset I$ is some interval including $\mu_{0}$. The following result is veiled in [R], and it is explicitly due to [TY] (using a setting in [GH]). Palis and Takens [PT] later independently used the same setting, getting to the same conclusion.

Proposition 3-7. For every $n \in \mathbb{N}$ there exists reparametrizations $\mu=M_{n}\left(\mu_{*}\right)$ of the $\mu$ variable, and $\mu_{*}$ dependent coordinate transformation $\left(x_{*}, y_{*}\right) \rightarrow \varphi_{n ; \mu_{*}}\left(x_{*}, y_{*}\right)$ such that

(1) for each compact set $K$ in the $\left(\mu_{*}, x_{*}, y_{*}\right)$-space, the images of $K$ under the maps $\left(\mu_{*}, x_{*}, y_{*}\right) \rightarrow\left(M_{n}\left(\mu_{*}\right), \varphi_{n ; \mu_{*}}\left(x_{*}, y_{*}\right)\right)$ converge for $n \rightarrow \infty$ to $\left(\mu_{0}, r_{0}\right)$;

(2) the domains of the maps $\left(\mu_{*}, x_{*}, y_{*}\right) \rightarrow\left(\mu_{*}, \varphi_{n ; \mu_{*}}^{-1} F_{M_{n}}^{n}\left(\mu_{*}\right) \circ \varphi_{n ; \mu_{*}}\left(x_{*}, y_{*}\right)\right)$ converge for $n \rightarrow \infty$ to all of $\mathbb{R}^{3}$, and the maps converge smoothly for $n \rightarrow \infty$ to the $\operatorname{map}\left(\mu_{*}, x_{*}, y_{*}\right) \rightarrow\left(\mu_{*}, \hat{F}_{\mu_{*}}\left(x_{*}, y_{*}\right)\right)$ with $\hat{F}_{\mu_{*}}\left(x_{*}, y_{*}\right)=\left(y_{*}, y_{*}^{2}-\mu_{*}\right)$.

Proof. For a proof, see [TY].

3D. Duration of KLUS-Simple Sinks and Attracting Sets. Before we define the duration of KLUS-simple sinks and attracting sets, we consider the family $\left\{f_{\beta}\right\}_{\beta \in[-1,3]}$ defined by $f_{\beta}(y)=y^{2}-\beta$. The following is well known:

(1) For $\beta \in\left[-1,-\frac{1}{4}\right) f_{\beta}^{n}(y) \rightarrow \infty$ as $n \rightarrow \infty$ for all $y$.

(2) For $\beta=-\frac{1}{4}$ a saddle node bifurcation occurs at $y_{0}=\frac{1}{2}$, for $\beta \in\left(-\frac{1}{4}, 3\right]$ there are two fixed points $v_{\beta}$ and $w_{\beta}$ such that $v_{\beta}$ is unstable and $w_{\beta}$ is a 1-sink (respectively unstable) if $\beta \in\left(-\frac{1}{4}, \frac{3}{4}\right)$ (respectively $\beta \in\left(\frac{3}{4}, 3\right]$ ).

(3) Write $I_{\beta}=\left[-v_{\beta}, v_{\beta}\right], J_{\beta}=\left[-\left(v_{\beta}+\beta\right) / 2,\left(v_{\beta}+\beta\right) / 2\right]$, and $A_{\beta}=w_{\beta}$ if $\beta \in\left(-\frac{1}{4}, 0\right]$ and $A_{\beta}=\left[f_{\beta}(0), f_{\beta}^{2}(0)\right]$ if $\beta \in(0,3]$; for $\beta \in\left[-\frac{1}{4}, 2\right] f_{\beta}\left(I_{\beta}\right) \subset I_{\beta} \subset[-2,2]$, for $\beta \in\left(-\frac{1}{4}, 2\right) A_{\beta}$ is a 1-attracting set and $w_{\beta} \in A_{\beta} \subset \operatorname{Int} J_{\beta} \subset J_{\beta} \subset \operatorname{Int} I_{\beta} \subset I_{\beta} \subset(-2,2)$, and $\bigcap_{n \geqq 0} f_{\beta}^{n}\left(J_{\beta}\right)=A_{\beta}$; for $\beta \in(2,3] \bigcap_{n \geqq 0} f_{\beta}^{n}\left(I_{\beta}\right)$ is a Cantor set.

We define the duration of the 1 -sink $w_{\beta}$ as the interval $\left(-\frac{1}{4}, \frac{3}{4}\right)$, and the duration of the 1-attracting set $A_{\beta}$ as the interval $\left(-\frac{1}{4}, 2\right)$. 
It is not difficult to show that for each $\beta \in\left(-\frac{1}{4}, 2\right)$ every two dimensional $C^{3}$-diffeomorphism, which is sufficiently $C^{3}$-close to $f_{\beta}$, also has a 1 -attracting set in the interior of $[-2,2] \times[-2,2]$. For an explanation, see [TY] (see also Sect. 4 for additional comments).

Let $H$ be a KLUS interval as in Sect. 3B. For $\mu_{0} \in H$, the duration in $\mathbf{H}$ of a KLUS-simple $p$-sink $z_{H ; \mu_{0}}^{p}$ is the component in $\left\{\mu \in H: z_{H ; \mu}^{p}\right.$ is KLUS-simple $p$-sink $\}$ that includes $\mu_{0}$; the duration in $\mathbf{H}$ of a KLUS-simple $p$-attracting set $\Gamma_{H ; \mu_{0}}^{p} S$ is the component in $\left\{\mu \in H: \Gamma_{H ; \mu}^{p}\right.$ is KLUS-simple $p$-attracting set $\}$ that includes $\mu_{0}$. We write $\operatorname{Dur}\left(\Gamma_{H}^{p}\right)$ (respectively $\left.\operatorname{Dur}\left(z_{H}^{p}\right)\right)$ for the union of the durations in $H$, and $\left|\operatorname{Dur}\left(\Gamma_{H}^{p}\right)\right|$ (respectively $\left.\mid \operatorname{Dur}\left(z_{H}^{p}\right)\right) \mid$ for the sum of the lengths of the durations in $H$ of all the $p$-attracting sets (respectively, $p$-sinks). Notice that the duration of a KLUS-simple $p$-attracting set $\Gamma_{\mu}^{p}$ equals the interval over which a topological horseshoe is formed for $F_{\mu}^{p}$ in a neighborhood $B$ in $S$ of the KLUS-simple $p$-path. The length of the interval is proportional to the length of the component in Proposition 3-5, because in order for $\Gamma_{\mu}^{p}$ to be attracting, the $L^{\text {th }}$ iterate of $B$ must be contained in the regions of Proposition 3-2.

The set of KLUS-simple parameter values in $H$ is, by definition, contained in $\bigcup_{k=p}^{\infty} \operatorname{Dur}\left(\Gamma_{H}^{k}\right)$ for every $p \in \mathbb{N}$. This implies that, for every $p$, the Lebesgue measure of the KLUS-simple parameter values is at most $\sum_{k \geqq p}\left|\operatorname{Dur}\left(\Gamma_{H}^{k}\right)\right|$. In order to obtain the result that the set of KLUS-simple parameter values in $H$ has Lebesgue measure zero, it is sufficient to show the following.

For each $\varepsilon>0$ there exists $P \in \mathbb{N}$ such that

$$
\sum_{k \geqq P}\left|\operatorname{Dur}\left(\Gamma_{H}^{k}\right)\right|<\varepsilon,
$$

where the sum is taken over all the KLUS-simple $p$-attracting sets for all $p \geqq P$, that arise (in $\mathrm{S}$ ) from the tangencies of all the saddles in the saddle basic sets $\left\{\Lambda_{\mu}: \mu \in H\right\}$.

Obviously, $\sum_{k \geqq P}\left|\operatorname{Dur}\left(\Gamma_{H}^{k}\right)\right|<\varepsilon$ implies that $\sum_{k \geqq P}\left|\operatorname{Dur}\left(z_{H}^{k}\right)\right|<\varepsilon$ (in the latter case we sum over all the KLUS-simple $p$-sinks for all $p \geqq P$ arising from all the tangencies of the saddles in the saddle basic sets $\left\{\Lambda_{\mu}: \mu \in H\right\}$ ).

3E. Proof of the Proposition. Let $I_{*} \subset I$ be a given interval and assume that $U$ is a saddle isolating neighborhood for the saddle basic set $\Lambda_{\mu}$, where $\mu \in I_{*}$. Assume that the family $\left\{F_{\mu}\right\}_{\mu \in I}$ creates for a $p$-saddle $q_{\mu} \in \Lambda_{\mu}$ a nondegenerate homoclinic intersection at $x=r_{0} \in M \backslash \operatorname{Clos} U, \mu=\mu_{0} \in \operatorname{Int} I_{*}$.

Select the minimal positive integers $K$ and $L$ such that $r_{0} \in F_{\mu_{0}}^{K}(U)$ and $r_{0} \in F_{\mu_{0}}^{-L}(U)$. Since $F_{\mu_{0}}^{K}(U) \cap F_{\mu_{0}}^{-L}(U)$ is open, there exists $\delta_{0}>0$ such that for each $\mu \in\left[\mu_{0}-\delta_{0}, \mu_{0}+\delta_{0}\right]^{\mu_{0}}$ the intersection $F_{\mu}^{K}(U) \cap F_{\mu}^{-L}(U)$ is open and it includes $r_{0}$. Let $\varepsilon>0$ such that $B\left(r_{0} ; \varepsilon\right) \subset \bigcap_{\mu \in H_{0}}\left(F_{\mu}^{K}(U) \cap F_{\mu}^{-L}(U)\right)$ and $B\left(r_{0} ; \varepsilon\right) \cap U=\varnothing$, where $B\left(r_{0} ; \varepsilon\right)$ the ball centered at $r_{0}$ with radius $\varepsilon$, and $H_{0}=\left[\mu_{0}-\delta_{0}, \mu_{0}+\delta_{0}\right]$. Define $S=B\left(r_{0} ; \varepsilon\right)$, and we obtain, by the definition of nondegenerate homoclinic bifurcation, that $H$ is a KLUS-interval, where $H=\left[\mu_{0}-\delta_{1}, \mu_{0}+\delta_{1}\right]$ for some $\delta_{1}$, $0<\delta_{1} \leqq \delta_{0}$. We conclude: the choice $d_{\mu_{0}}=\delta_{1}$ gives the desired result. This completes the proof of the proposition. 
3F. Proof of the Theorem. Let the compact interval $I$ be given. For each $\mu \in I$, let $\Lambda_{\mu}$ be the saddle basic set that varies differentiably with $\mu$, and let $V_{\Lambda ; \mu}^{\mathrm{s}}$ be a neighborhood of $\Lambda_{\mu}$ on which the stable foliation $\mathfrak{F}_{\mu}^{s}$ is defined. We will assume that $F_{\mu}$ has for every $k \in \mathbb{N}$ and for each $\mu \in I$, only either finitely many or no periodic points with period $k$; this assumption is no restriction, see Yorke and Alligood [YA].

We may assume that $U$ is a saddle isolating neighborhood for the saddle basic set $\Lambda_{\mu}$ for all $\mu \in I$, because of the differentiable dependence of $\Lambda_{\mu}$ on $\mu$, there are finitely many compact subintervals, say $I_{1}, \ldots, I_{Q}$ of $I$, and for each $k$ there is saddle isolating neighborhood $U_{k}$ of $\Lambda_{\mu}$ for $\mu \in I_{k}$, and on which the following applies.

Assume that the family $\left\{F_{\mu}\right\}_{\mu \in I}$ creates a nondegenerate homoclinic intersection at $x=r_{0} \in M \backslash \operatorname{Clos} U, \mu=\mu_{0} \in \operatorname{Int} I$ for a $p$-saddle $q_{\mu} \in \Lambda_{\mu}$. Select the minimal positive integers $K, L$ such that $r_{0} \in F_{\mu_{0}}^{K}(U) \cap F_{\mu_{0}}^{-L}(U)$. Select $\delta_{0}>0$ such that for each $\mu \in\left[\mu_{0}-\delta_{0}, \mu_{0}+\delta_{0}\right]=H_{0}$ the interior of the intersection $\bigcap_{\mu \in H_{0}}\left[F_{\mu}^{K}(U) \cap F_{\mu}^{-L}(U) \cap\right.$ $\left.F_{\mu}^{-L}\left(V_{\Lambda ; \mu}^{s}\right)\right]$ is nonempty, and includes $r_{0}$. Let $\varepsilon_{0}>0$ such that $B\left(r_{0} ; \varepsilon_{0}\right) \subset$ Int $\bigcap_{\mu \in H_{0}}\left[F_{\mu}^{K}(U) \cap F_{\mu}^{-L}(U) \cap F_{\mu}^{-L}\left(V_{\Lambda ; \mu}^{s}\right)\right]$, and $B\left(r_{0} ; \varepsilon_{0}\right) \cap U=\varnothing$, where $B\left(r_{0} ; \varepsilon_{0}\right)$ the ball centered at $r_{0}$ with radius $\varepsilon_{0}$. Define $S=B\left(r_{0} ; \varepsilon_{0}\right)$, and select $\delta_{1}, 0<\delta_{1} \leqq \delta_{0}$ so that $H=\left[\mu_{0}-\delta_{1}, \mu_{0}+\delta_{1}\right]$ satisfies all the conditions of a KLUS interval, see Sect. 2 for details.

From the definition of $S$, we know that the stable foliation $\mathfrak{F}_{\mu}^{s}$ can be extended to exist on $S$ for every $\mu \in H$. Therefore, from now on, we will assume that the stable foliation $\mathfrak{F}_{\mu}^{s}$ exists on $S$, for all $\mu \in H$.

Recall that for $\mu_{0} \in H$, the duration in $H$ of a KLUS-simple $p$-sink $z_{H ; \mu_{0}}^{p}$ is the component in $\left\{\mu \in H: z_{H ; \mu}^{p}\right.$ is KLUS-simple $p$-sink $\}$ that includes $\mu_{0}$; the duration in $H$ of a KLUS-simple $p$-attracting set $\Gamma_{H ; \mu_{0}}^{p}$ is the component in $\left\{\mu \in H: \Gamma_{H ; \mu}^{p}\right.$ is KLUS-simple $p$-attracting set $\}$ that includes $\mu_{0}$. We write $\operatorname{Dur}\left(\Gamma_{H}^{p}\right)$ (respectively $\left.\operatorname{Dur}\left(z_{H}^{p}\right)\right)$ for the union of the durations in $H$, and $\left|\operatorname{Dur}\left(\Gamma_{H}^{p}\right)\right|\left(\operatorname{respectively} \mid \operatorname{Dur}\left(z_{H}^{p}\right)\right) \mid$ for the sum of the lengths of the durations in $H$ of all the $p$-attracting sets (respectively, $p$-sinks). As explained in Sect. 3D, the set of KLUS-simple parameter values in $H$ has Lebesgue measure zero if for each $\varepsilon>0$ there exists $P \in \mathbb{N}$ such that

$$
\sum_{k \geqq P}\left|\operatorname{Dur}\left(\Gamma_{H}^{k}\right)\right|<\varepsilon,
$$

where the sum is taken over all the KLUS-simple $p$-attracting sets for all $p \geqq P$, that arise (in $S$ ) from the tangencies of all the saddles in the saddle basic sets $\left\{\Lambda_{\mu}: \mu \in H\right\}$.

Let $\delta_{\Lambda ; H}>0$ as in Sect. 3B. Let $\varepsilon>0$ be given; applying Proposition 3-5, Proposition 3-7, Lemma 3-6, and the fact that the stable foliation on $S$ exists and the projection along the stable manifolds of $\Lambda_{\mu}$ is $C^{1+\alpha}$ yields there exists a constant $C_{0}>0$ such that for each $p \geqq Q \gg K+L$ the total length of the duration in $H$ of all the KLUS-simple $p$-attracting sets is at most $C_{0} \cdot\left[1-\delta_{\Lambda ; H}\right]^{2(p-K-L)}=$ $C_{1} \cdot\left[1-\delta_{\Lambda ; H}\right]^{2 p}$, where $C_{1}=C_{0} \cdot\left[1-\delta_{\Lambda ; H}\right]^{-2(K+L)}$.

Select integer $P \geqq Q$ such that $C_{1} \cdot\left[1-\delta_{\Lambda ; H}\right]^{-2} \cdot\left[1-\delta_{\Lambda ; H}\right]^{2 P}<\varepsilon$. Then, for the 
total duration in $H$ of all the $k$-attracting sets for all $k \geqq P$ we get

$$
\begin{aligned}
\sum_{k \geqq P}\left|\operatorname{Dur}\left(\Gamma_{H}^{k}\right)\right| & \leqq \sum_{k \geqq P} C_{1} \cdot\left[1-\delta_{\Lambda ; H}\right]^{2 k} \\
& =C_{1} \cdot\left[1-\delta_{\Lambda ; H}\right]^{2 P} \cdot \sum_{k \geqq 0}\left[1-\delta_{\Lambda ; H}\right]^{2 k} \\
& \leqq C_{1} \cdot\left[1-\delta_{\Lambda ; H}\right]^{2 P} \cdot\left(\sum_{k \geqq 0}\left[1-\delta_{\Lambda ; H}\right]^{k}\right)^{2} \\
& =C_{1} \cdot\left[1-\delta_{\Lambda ; H}\right]^{2 P} \cdot\left[1-\delta_{\Lambda ; H}\right]^{-2}<\varepsilon .
\end{aligned}
$$

The conclusion is that the set of KLUS parameter values in $H$ for the above $S$ is zero.

For each nondegenerate tangency value $\mu_{0} \in I$, for which $\left\{F_{\mu}\right\}_{\mu \in I}$ creates a nondegenerate homoclinic intersection at $x=r_{0} \in M \backslash \operatorname{Clos} U$ for a $p$-saddle $q_{\mu} \in \Lambda_{\mu}$, the above applies. Since both $M \backslash U$ and $I$ are compact, we conclude that the set of all KLUS parameter values in $I$ is zero. This completes the proof of the theorem.

\section{Discussion and Concluding Remarks}

4-1. Order of Differentiability of the Diffeomorphism. We assume that the diffeomorphism $F$ is $C^{3}$. This assumption implied the existence of a $C^{1+\alpha}$ expanding map, for some $\alpha>0$, in Proposition 3-3. If $F$ is of class $C^{2}$, then it is known that such an expanding map is $C^{1}$. We would like to point out that the Hölder exponent $\alpha$ is only used to obtain (2) in the proof of Proposition 3-5. Fortunately, we can prove Proposition 3-5 (in particular property (2)) for the $\mathrm{C}^{1} \operatorname{map} \varphi$ of Proposition $3-3$ by combining the techniques of the proof of Proposition 6 in $[\mathrm{Ne}]$ and Lemma 5.5 in $[\mathrm{Nu}]$. Thus in fact, it is sufficient to assume $F$ is $C^{2}$ to guarantee the main results of the paper.

4-2. Non-Simple Attractors. Consider a KLUS-simple $p$-sink. In [TY] it is shown that these sinks, for $p$ large enough, exist by proving that a $p$-sink is created near a nondegenerate tangency value via a saddle node bifurcation at $\mu_{p}$. The family $F_{\mu}^{p}$ is $C^{3}$-close to the quadratic map of the interval, both in space and parameter. The parameter interval $I_{p}$ containing $\mu$, and the neighborhood $\operatorname{Box}_{p} \subset \mathbb{R}^{2}$ of the $p$-sink obey a rescaling law as $p \rightarrow \infty\left(\left|I_{p}\right| \propto \lambda^{-2 p}\right.$, where $\lambda>2$ is the eigenvalue of the affine horsehoe). For each possible structure of a periodic orbit with period $n=k \cdot p$ there exists a parameter value $\mu_{k} \in I_{p}$ at which such an orbit is created in Box $_{\mathrm{p}}$. In fact, a topological horsehoe is created for $F_{\mu}^{p}$ as $\mu$ varies through $I_{p}$. Therefore, since $F_{\mu}^{p}$ is dissipative, each of these periodic orbits of period $k \cdot p$ is a sink for some $\mu_{k} \in I_{p}$ (for a proof, see [YA]). This is a subclass of non-simple KLUS-sinks (when $k \geqq 2$ ).

We call the KLUS-simple $p$-sink the pioneer of such a topological horseshoe. For each $p$, the associated $n$-sinks with $n=k \cdot p$ appear as $\mu$ varies in $I_{p^{\prime}}$ in an order that tends (as $p \rightarrow \infty$ ) to the Sarkovski order of the map of the interval. In particular, given $k_{1}, k_{2} \in \mathbb{N}$, for each $p$ sufficiently large, the corresponding $n_{1}$-sink and $n_{2}$-sink (with $n_{1}=k_{1} \cdot p$ and $n_{2}=k_{2} \cdot p$ ) do not coexist. 
However, for fixed $p$, in the process of the formation of a topological horseshoe, the KLUS-simple $p$-saddle forms tangencies, and therefore the Newhouse phenomenon of infinitely many coexisting sinks holds. That is, the above $n$-sinks can and do coexist for finite $p$. Holmes and Whitley [HW] nicely rephrased the Newhouse phenomenon as an overlapping of the $p$-duration of sinks in two-parameter families $F_{\mu ; \varepsilon}: \mathbb{R}^{2} \rightarrow \mathbb{R}^{2}$, where the parameter $\varepsilon$ is a distance of the family $F_{\mu ; \varepsilon}$ from the one dimensional quadratic map.

Of the above $n$-sinks some never coexist (for example, $p \cdot 2^{k}$-sinks which form a "cascade") and their duration is already accounted for in the Duration of the $p$-attracting set. For the others, one can copy all of our arguments for the pioneer KLUS-simple $p$-saddle forming tangencies and prove that there are sinks which are "simple" with respect to the pioneer, so they are simple at a second hierarchial level. Infinitely many of them can therefore coexist only on a parameter set of measure zero. Since under generic assumptions, there are countably many pioneers, such KLUS-simple sinks of second level yield a measure zero set of KLUS-simple second level Newhouse parameter values.

4-3. The [TY]-Simple Newhouse Parameter Values. Comparing with the results in [TY], we have a generalization for saddle hyperbolic basic sets, in particular, a generalization for nonlinear horseshoe maps. Notice that in [TY] an "attracting set" was called an "attractor."

4-4. Dimension. For clarity, we stated the theorem in Lebesgue measure. However, anyone who is familiar with dimensions, will observe that the proof of the theorem implies that the Box counting dimension (that is, the Capacity dimension) of the set of KLUS-simple parameter values is less than one, which is slightly more general.

Acknowledgements. A great part of this paper was written while H. E. N. was on the GNFM-CNR program for visiting professors at the University of Rome "La Sapienza." L.T.L. wishes to acknowledge useful conversations with Sheldon Newhouse in the preliminary stages of this work.

\section{References}

[GH] Guckenheimer, J., Holmes, P.: Nonlinear oscillations, Dynamical systems, and bifurcations of vector fields. Applied Mathematical Sciences Vol. 42. Berlin, Heidelberg, New York: Springer 1983

[HW] Holmes, P. J., Whitley, D.: Bifurcations of one and two dimensional maps. Phil. Trans. Royal Society London A 311, 43-102. Erratum 312, 601-602 (1984)

[NP] Newhouse, S., Palis, J.: Hyperbolic nonwandering sets on two-dimensional manifolds. In: Dynamical Systems, pp. 293-301, Peixoto, M. M. (ed.). New York, London: Academic Press 1973

[Ne1] Newhouse, S. E.: Diffeomorphisms with infinitely many sinks. Topology 13,9-18(1974)

[Ne2] Newhouse, S. E.: The abundance of wild hyperbolic sets and non-smooth stable sets for diffeomorphisms. Publ. Math. I.H.E.S. 50, 101-151 (1979)

$[\mathrm{Nu}]$ Nusse, H. E.: Asymptotically periodic behaviour in the dynamics of chaotic mappings. SIAM J. Appl. Math. 47, 498-515 (1987)

[NY] Nusse, H. E., Yorke, J. A.: Analysis of a procedure for finding numerical trajectories close to chaotic saddle hyperbolic sets. Ergodic Theory Dyn. Syst. 11, 189-208 (1991)

[PR] Patterson, S., Robinson, C.: Basins of sinks near homoclinic tangencies. In: Dynamical Systems and Bifurcation Theory. Camacho, M. I., Pacifico, M. J., Takens, F. (ed.) pp. 
347-376. Pitman Research Notes in Mathematics Series, Vol. 160. London: Longman Scientific \& Technic 1987

[PT] Palis, J., Takens, F.: Homoclinic bifurcations and hyperbolic dynamics. $16^{\circ}$ Colóquio Brasileiro Matemática, IMPA 1987 and Cambridge University Press, to appear 1992

[R] Robinson, C.: Bifurcations to infinitely many sinks. Commun. Math. Physics 90, 433-459 (1983)

[TY] Tedeschini-Lalli, L., Yorke, J. A.: How often do simple dynamical processes have infinitely many coexisting sinks? Commun. Math. Physics 106, 635-657 (1986)

[W] Wang, X.-J.: The Newhouse set has a positive Hausdorff dimension. Commun. Math. Physics 131, 317-332 (1990)

[YA] Yorke, J. A., Alligood, K. T.: Period doubling cascades of attractors: A prerequisite for horseshoes. Commun. Math. Phys. 101, 305-321 (1985)

Communicated by J. N. Mather 